\title{
The Effects of Tyrosine-Protein Kinase Kit on Bronchopulmonary Dysplasia
}

\author{
Lan Guofeng ${ }^{1}$, Wang Yijin ${ }^{1}$, Li Yunfang ${ }^{1}$, Wei Qinghua ${ }^{1}$, Shi Fenglang ${ }^{1}$, Cui Qiliang ${ }^{2}$, Hussnain Mirza $^{3}$ and $^{-}$ \\ Shi Xuekai ${ }^{1 *}$ \\ ${ }^{1}$ Department of Pediatrics, the Second People's Hospital of Nanning, NO.13, Dancun Road, Jiangnan District, Nanning, China \\ ${ }^{2}$ The Third Affiliated Hospital of Guangzhou Medical University, NO.63,Duobao Road, Liwan District, Guangzhou, China \\ ${ }^{3}$ Florida Hospital for Children, Center for Neonatal Care, Florida children's Hospital, USA
}

*Corresponding author: Shi Xuekai, Department of Pediatrics, the Second People's Hospital of Nanning, N0.13, Dancun Road, Jiangnan District, Nanning, 530031, China.

\begin{abstract}
Bronchopulmonary dysplasia (BPD) is a multifactorial chronic pulmonary disorder which complicates multiple pulmonary hypertensions in preterm infants. At present, there are no effective prevention or treatment options for BPD in clinical practice. Tyrosine-Protein Kinase Kit (KIT) serves an important role in regulating cell proliferation, hematopoiesis and stem cell maintenance. In the present study, the protective role of overexpression of KIT in BPD was investigated. The candidate differentially expressed genes (DEGs) between patients with BPD and healthy controls were screened using bioinformatics. A neonatal BPD mouse model was established under a hyperoxic environment and KIT overexpressing cells were intravenously injected into the mice, followed by evaluation of the effects on respiratory system resistance, pulmonary development and remodeling. Bioinformatics analysis showed that KIT was down regulated in patients with BPD, and a protein-protein interaction network was created using the Search Tool for Recurring Instances of Neighboring Genes database, which indicated that KIT was associated with known diseaserelated genes and regulated VEGF expression. In neonatal BPD mice, KIT exhibits significant protective affects and may thus serve as a candidate therapeutic target for treatment of patients with BPD treatment
\end{abstract}

Keywords: Bronchopulmonary dysplasia; Tyrosine-Protein Kinase Kit; Vascular endothelial growth factor; Pulmonary fibrosis, type II collagen; CD31

\section{Introduction}

Bronchopulmonary dysplasia (BPD) is a common chronic disorder of the respiratory system in preterm infants, particularly in very premature infants. BPD is characterized by alveolar dysplasia and a reduced number of alveoli, alveolar simplification, capillary dysplasia and other symptoms [1]. The incidence of BPD is $12-32 \%$ in preterm infants at $<32$ weeks of gestation [2] and may rise to as high as $50 \%$ in infants with extremely low birth weights $(<1,000 \mathrm{~g})$ or extremely low gestational age ( $<28$ weeks) [3]. The incidence of BPD has been increasing for years and the lack of effective therapeutic measures has severely affected the outcomes and survival of BPD infants. Epidemiological studies have shown that BPD is a complex disease and many factors, including oxygen exposure time, mechanical ventilation and strength, inflammatory reactions, lung tissue immaturity, damage and other factors may lead to BPD [4]. Additionally, studies have shown that stem cells may effectively interfere with the development of BPD [5,6].

Tyrosine-Protein Kinase Kit (KIT) is a transmembrane protein receptor associated with germ cell maturation [7] and is encoded by the human homolog of the proto- oncogene c-kit. It serves an important role in regulating cell proliferation, hematopoiesis and 
stem cell maintenance. KIT activation has been shown to exhibit oncogenic activity in gastrointestinal stromal tumors (GISTs), melanomas, lung cancer, and other types of tumors. The targeted therapeutics, nilotinib and sunitinib, exhibit efficacy in treating KIT overactive patients in late-stage trials in patients with melanoma and GIST. KIT over activity may be the result of numerous genomic events, including genomic amplification, overexpression and missense mutations. Missense mutations have been demonstrated to be key players in mediating clinical response and acquired resistance in patients being treated with this targeted therapeutics. Furthermore, Kit ligand polymorphisms are associated with susceptibility to moderate- to-severe BPD [8-10].

Vascular endothelial growth factor (VEGF) is a central factor in angiogenesis and its expression levels affect pulmonary vascular development, consequently impacting the development and progression of BPD [11]. VEGF is primarily produced by alveolar epithelial cells and exerts effects on endothelial cell migration, survival, proliferation, and differentiation, and it is an essential regulatory factor required for the growth and maintenance of pulmonary vasculature during the embryonic, fetal and postnatal stages [12]. Deficiency of VEGF may affect the formation of a normal fetal pulmonary capillary system, leading to reduced capillary bed density, alveolar simplification and impaired alveolar development [13], thereby increasing the incidence of BPD in neonates. These observations demonstrate the essential role of VEGF in maintaining normal lung development. Additionally, pulmonary angiogenesis is strongly influenced by VEGF-A. VEGF-A is a specific mitogen and survival factor in vascular endothelial cells, and it is expressed by distal airspace epithelial cells in both the fetal and postnatal lung [14]. Common polymorphisms of the gene encoding VEGF-A are associated with lung function in both children and in adults [15].

Based on previous studies, a comprehensive analysis strategy was used on the GSE25286 gene expression profile obtained from Gene Expression Omnibus (GEO), the associations between VEGF expression, disease-related genes and differentially expressed genes (DEGs) were determined, and a protein-protein interaction (PPI) network was constructed. Additionally, in a mouse model of BPD, the effects of KIT expression on the maintenance of pulmonary vascular formation and alveolar growth were evaluated and the underlying mechanisms were studied. The results of these experiments demonstrated the protective effects of KIT on BPD and highlight KIT as a candidate therapeutic target for the treatment of BPD.

\section{Materials and Methods}

\section{Bioinformatics analysis}

The GSE25286 profile and the corresponding platform annotation files were obtained from the GEO database (ncbi.nlm. nih.gov/geo/query/acc.cgi?acc=GSE25286). A Limma Microarray/
Counts test for DEGs was performed, and genes with fold changes (FCs) $>1$ and an adjusted $\mathrm{P}<0.05$ were considered further.

The DEGs between BPD and control lung tissues, with high or low mRNA expression levels were computed using the R package "limma". In total, DEGs with an absolute $\log 2 \mathrm{FC} \geq 1$ and an adjusted $\mathrm{P}<0.05$ were considered analyzed further using univariate Cox regression analysis. Adjusted P-values for multiple tests were determined using Benjamini-Hochberg correction.

The PPI network was generated using the Search Tool for Recurring Instances of Neighboring Genes (STRING) database (string-db.org) and Cytoscape software was used to create the images based on the STRING results.

\section{Establishment of a hyperoxia-induced BPD model}

A total of 9 newborn FVB mice, a common genetic research model which was not particularly relevant in hyperoxia studies and 2 mothers were exposed to $75 \%$ oxygen in an organic glass chamber or normal indoor air at the time of birth and reared continuously for 14 days. An oxygen concentration controller (BioSpherix, Ltd.) was used to adjust air flow, maintain the oxygen concentration in the chamber at $75 \%$, and remove $\mathrm{CO} 2$, ensuring that $\mathrm{CO} 2$ concentration never exceeded $0.5 \%$. Ammonia was filtered through an air purifier and activated charcoal. Normoxic and hyperoxic mother mice were swapped every $48 \mathrm{~h}$ to prevent oxygen toxicity in mother mice.

For the hyperoxic group ( $n=3)$, the newborn FVB mice were reared in $75 \%$ oxygen conditions for 2 weeks starting from postnatal day 1 (within $6 \mathrm{~h}$ of birth). For the normoxic group $(n=6)$, the newborn FVB mice were reared in normal room air for 2 weeks starting from birth. When chronic hyperoxia injury became obvious in the BPD model on day 14 [16], mice were placed in normal room air, and the hyperoxic mice were randomly divided into three groups as follows: Hyperoxia model group $(n=2)$, hyperoxia model + negative control $(\mathrm{NC})$ group $(\mathrm{n}=2)$, and hyperoxia model + KIT group $(n=2)$. The normoxia group and hyperoxia model group were placed in normal room air and reared for 2 weeks. The hyperoxia model $+\mathrm{NC}$ group were intravenously/intramuscularly injected with NC lentivirus, and the hyperoxia model + KIT group were intravenously/intramuscularly injected with KIT overexpression lentivirus; both groups were placed in normal room air and reared for 2 weeks. Each group of neonatal mice was allowed to recover for 2 weeks and subsequently, the mice were anesthetized with chloral hydrate $(400 \mathrm{mg} / \mathrm{kg})$ and sacrificed by cervical dislocation. Lung tissues were collected for histological and immunohistochemical studies as described below.

Mice were housed under controlled environmental conditions with free access to water and food, and 12-hour alternating light/ dark cycles. Animal care and handling were conducted according to the guidelines of the Medical Ethics Committee and Institutional Review Board of the Second People's Hospital of Nanning, China. 


\section{Measurement of respiratory system resistance}

To evaluate respiratory system resistance in newborn mice, an ultrasound nebulizer (SCIREQ Scientific Respiratory Equipment, Inc) was used to nebulize normal saline and methacholine (1.6, 5, 10,16 , or $50 \mathrm{mg} / \mathrm{ml}$ ) for inhalation. Mean airway resistance was calculated at the baseline time, and the maximum value for each methacholine dose was subsequently recorded.

Masson's trichrome staining. Alveolar tissue was stained using Masson's trichrome stain. Alveolar cells were dehydrated and embedded for paraffin sectioning. The nucleus was stained using hematoxylin for $15 \mathrm{~min}$ and then ponceau $\mathrm{S}$ acid solution for 10 min. Slides were treated with phosphomolybdic acid solution for 5 min, counterstained in aniline for $5 \mathrm{~min}$, dehydrated multiple times, and mounted. Alveolar tissue fibrosis and the status of alveolar remodeling were analyzed in each group.

\section{Immunohistochemistry}

Lung tissue was dehydrated and embedded for sectioning. Sections were incubated with CD31 primary antibody (rabbit anti-mouse; 1:100; cat. no. 77699; Cell Signaling Technology, Inc.), transforming growth factor (TGF)- $\beta$ primary antibody (rabbit antimouse; 1:100; Sigma-Aldrich; Merck KGaA; cat. no. SAB4504269), type II collagen (COL II) primary antibody (rabbit anti-mouse, 1:100 dilution; Sigma-Aldrich; Merck KGaA; cat. no. SAB4500362), or type $\mathrm{V}$ collagen (COL V) primary antibody (rabbit anti-mouse; 1:100 dilution; Abcam; cat. no. ab7046) overnight at $4^{\circ} \mathrm{C}$. Goat anti-rabbit secondary antibody was used at a dilution of 1:1000 for $30 \mathrm{~min}$ at room temperature. Color was developed by staining with 3,3'Diaminobenzidine for $30 \mathrm{sec}$, and hematoxylin counterstaining was performed for $15 \mathrm{~min}$. The slides were dehydrated and mounted for immunohistochemical analysis.

\section{Lentivirus and KIT overexpression}

The cDNA sequence of KIT was obtained from GenBank (NM_000222.2). The coding region of KIT was obtained by PCR using primers (forward: 5'- CAGCTACCGCGATGAGAG-3'; reverse: 5'- GGGATTTATATATGTACATTTTATTAG AAT-3') and then inserted into the pLVX- shRNA1 vector (Clontech Corporation) using the BamHI and EcoRI restriction sites. Lentiviruses were generated using the pLVX-shRNA1 vector containing the coding sequence of KIT. Lentivirus was intravenously/intramuscularly administrated to the mice and the number of viral particles administered to each mouse was $1 \times 106$.

mRNA expression levels of KIT and VEGF. Lv-KIT (KIT overexpression vector) and short hairpin (sh)-VEGF (VEGF knockdown vector) were transfected into cells, either alone or together, to determine the effects of VEGF on functional changes in the lung caused by KIT overexpression. The mRNA expression levels of KIT and VEGF were measured by RT-qPCR in 293T cells, which was used as the intermediate cell-line for gene reconstruction. Fulllength cDNA encoding human VEGF was amplified from first-strand cDNA derived from the 293T cell line with an RNeasy plus mini kit (QIAGEN), High Capacity cDNA Reverse Transcription Kit (Applied Biosystems), Phusion HF DNA polymerase (Finnzymes).

Reverse transcription-quantitative (RT-q) PCR. Gene expression in whole lungs tissues were analyzed using RT-qPCR with specific primers (Applied Biosystems; Thermo Fisher Scientific, Inc.). Total RNA was extracted using an RNeasy Mini kit (Qiagen, Inc). qPCR was performed using Real Time TaqMan on an ABI Prism 7700 sequence detection system. The sequences of the primers were as follows: KIT forward, 5'- GCACAATGGCACGGTTGAAT-3' and reverse, 5'-GGTGTGGGGATGGATTTGCT- 3'; VEGF-A forward, 5'-CTCTCTCTCCCAGATCGGTGA-3' and reverse, 5'- CAAAGGAATGTGTGGTGGGGA-3'; and GAPDH forward, 5'- TTCCACCTTTGATGCTGGGG-3', and reverse, 5'-CCACCACCCTGTTGCTGTAG-3'. GAPDH was used as the internal control.

\section{Statistical analysis}

Data are presented as the mean \pm standard deviation using SPSS version 17.0 (SPPS, Inc). Statistical significance between $>2$ groups was determined using bonfferoni after a one-way ANOVA analysis, and a t-test was used to compare two groups. $\mathrm{P}<0.05$ was considered to indicate a statistically significant difference.

\section{Results}

KIT expression is down regulated in hyperoxia induced BPD mice. DEGs between BPD and control lung tissues were determined by obtaining the GSE25286 dataset from GEO, and bioinformatics tools were used to analyze this dataset. The results indicated that KIT expression was relatively lower in BPD samples compared with the controls (Figure 1A and B). Furthermore, the association between expression of the VEGF genes (PGF, VEGFA, VEGFB and VEGFC), disease-related genes (EPAS1, POSTN, IL1B, ACE, MMP2, HIF1A, TNF, TXN, SFTPD and SFTPC) and differentially expressed genes (ADM, TYMS, KIT, CD37, CD79B, CD22 and MS4A1) were determined using PPI networks. The results showed that KIT was closely associated with VEGF-A, and known disease-related genes in BPD tissues (Figure 1C). Based on the STRING database, it was demonstrated that VEGF-A was a downstream target gene of KIT (Figure 1D) [17] and VEGF expression was down regulated in BPD as well as key genes associated with repair in BPD [18-21]. Therefore, the functional effects of overexpression of KIT and its effects on VEGF-mediated repair in BPD were investigated. 

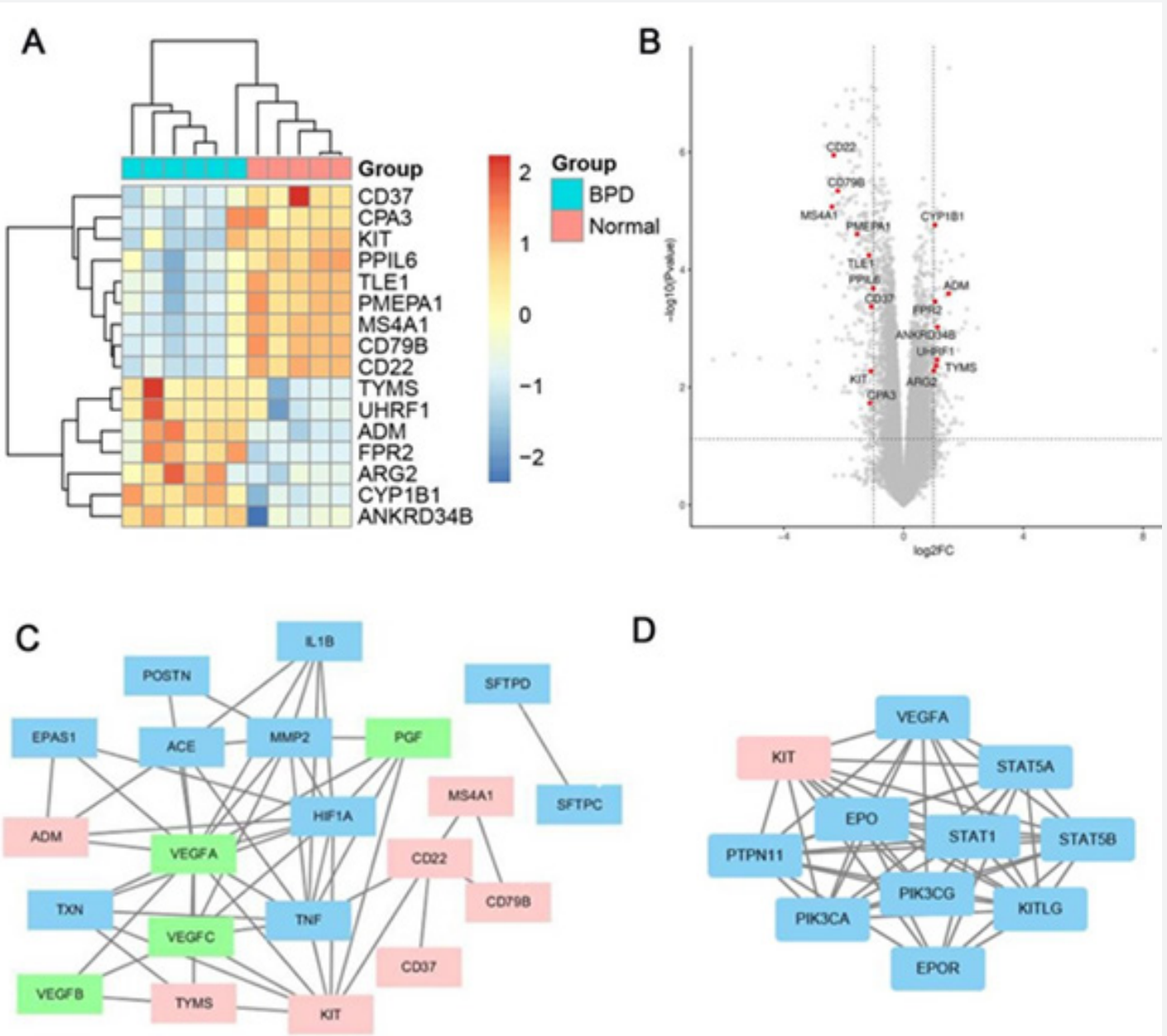

D

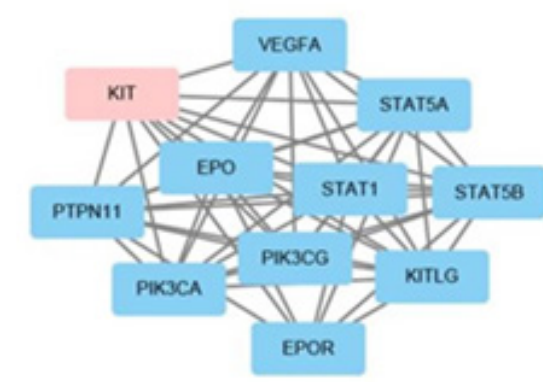

Figure 1: KIT expression is decreased in hyperoxia induced BPD mice. (A) Heat maps of the DEGs between control and BPD lung tissues in the GSE25286 dataset. (B) Volcanic maps of DEGs in the GSE25286 dataset. (C) Protein-protein interaction network of the DEGs (red; ADM, TYMS, KIT, CD37, CD79B, CD22, MS4A1); VEGF (green; PGF, VEGFA, VEGFB, VEGFC) and disease-related genes (blue; EPAS1, POSTN, IL1B, ACE, MMP2, HIF1A, TNF, TXN, SFTPD, SFTPC). (D) Interaction network diagram of KIT and VEGF. KIT, Tyrosine-Protein Kinase Kit; BPD, Bronchopulmonary dysplasia DEGs, differentially expressed genes.

KIT overexpression improves respiratory system resistance, alveolar remodeling and pulmonary fibrosis in BPD mice. To investigate whether KIT overexpression resulted in initiation of repair processes in BPD, a KIT overexpression lentivirus was constructed, and the overexpression efficiency of KIT in 293T cells was confirmed by RT-qPCR (Figure 2A).

To determine the effect of KIT overexpression on respiratory system resistance in the BPD model mice, an ultrasound nebulizer was used to nebulize normal saline and $50 \mathrm{mg} / \mathrm{ml}$ methacholine for inhalation by newborn mice [22]. As shown in Figure 2B, airway resistance was significantly higher in the hyperoxia-induced BPD group (Hyperoxia group) compared with the control group, and this affected lung development. Similar to a previous study by Lui et al. [23], mice with extremely low birth weights which subsequently developed BPD, exhibited higher respiratory system resistance. Respiratory system resistance was significantly reduced in KIT- overexpressing hyperoxia-induced BPD mice (KIT-hyperoxia) compared with the BPD model mice and NC-hyperoxia group (Figure 2B). Together, these data suggest that KIT overexpression in BPD may significantly reduce respiratory system resistance in BPD mice.

The hyperoxia and NC-hyperoxia groups showed notably drastic alterations in overall alveolar structure, tracheal deformation, alveolar simplification, airway remodeling, infiltration of inflammatory cells, increased collagen content in lung cells, and severe pulmonary fibrosis compared with the control group. In contrast, the KIT- hyperoxia group showed significantly improved alveolar injury structure, reduced levels of pulmonary fibrosis, decreased collagen content, abundant and organized alveolarization and normal airway structures compared with the hyperoxia group (Figure 2C). Therefore, KIT upregulation reduced hyperoxia-BPD induced alveolar structural abnormalities. 
A

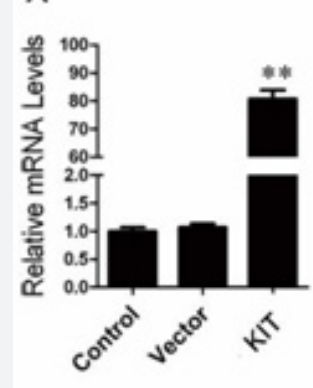

B

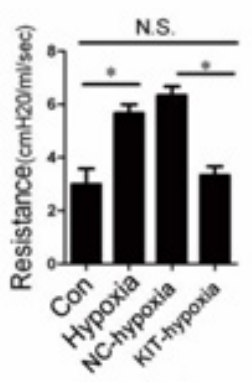

C
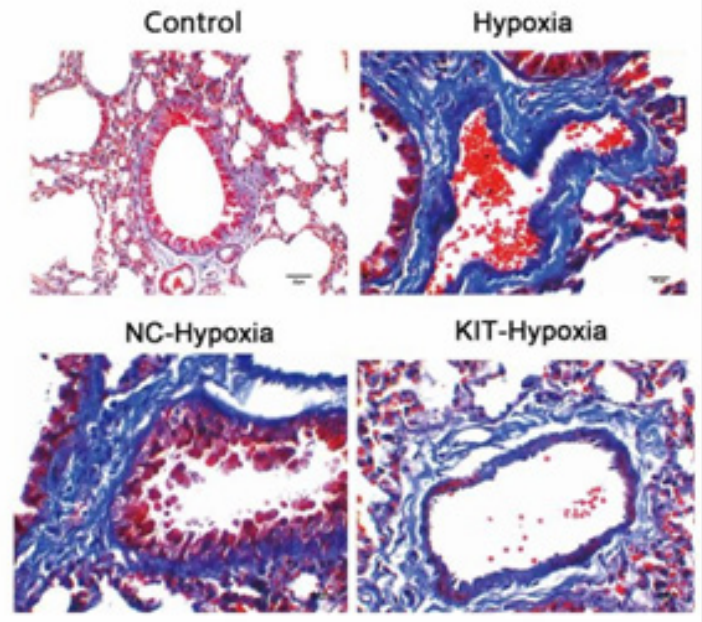

Figure 2: KIT overexpression improves respiratory system resistance, alveolar remodelling and pulmonary fibrosis. (A) KIT expression in the overexpression group was significantly higher compared with the control group. (B) Following KIT- overexpression in the BPD model in newborn mice, respiratory system resistance was decreased to levels similar to those observed in the normal healthy newborn mice. ${ }^{* *} \mathrm{P}<0.01,{ }^{*} \mathrm{P}<0.05$. (C) Determination of alveolar remodelling using Masson's trichrome staining. Lung section staining $(<100 \mu \mathrm{m})$ showed that the lung structure and composition in hyperoxia newborn mice was severely altered and pulmonary fibrosis was observed (blue staining). Overexpression of KIT prevented these structural and compositional changes. Magnification, x400 (D) Western blot analysis of Ki67 protein expression levels showed that Ki67 expression in the KIT + hyperoxia group was significantly increased and were similar to the levels observed in the normal healthy newborn mice. KIT, Tyrosine-Protein Kinase Kit, BPD, Bronchopulmonary dysplasia.

KIT overexpression reverses changes in the expression levels of angiogenesis and fibrosis-associated proteins in BPD mice. To determine the role of KIT on angiogenesis, immunohistochemistry analysis of BPD pulmonary fibrosis tissues was performed to determine the expression of the angiogenesis-associated protein, CD31, and the fibrosis-associated proteins, TGF- $\beta$, COL II and COL V. The results showed that CD31 expression was significantly reduced and TGF- $\beta$, COL II and COL V expression were significantly increased in the hyperoxia group and NC-hyperoxia group compared with the control group. However, in the KIT hyperoxia group, CD31 protein expression levels were increased, whereas the expression levels of TGF- $\beta$, COL II and COL V were significantly decreased compared with the hyperoxia and NC-hyperoxia groups (Figure 3). This observation suggests that KIT may promote angiogenesis through the upregulation of CD31 protein expression and inhibit pulmonary fibrosis by decreasing the protein expression levels of TGF- $\beta$, COL II and COL V, consistent with a previous study [24].

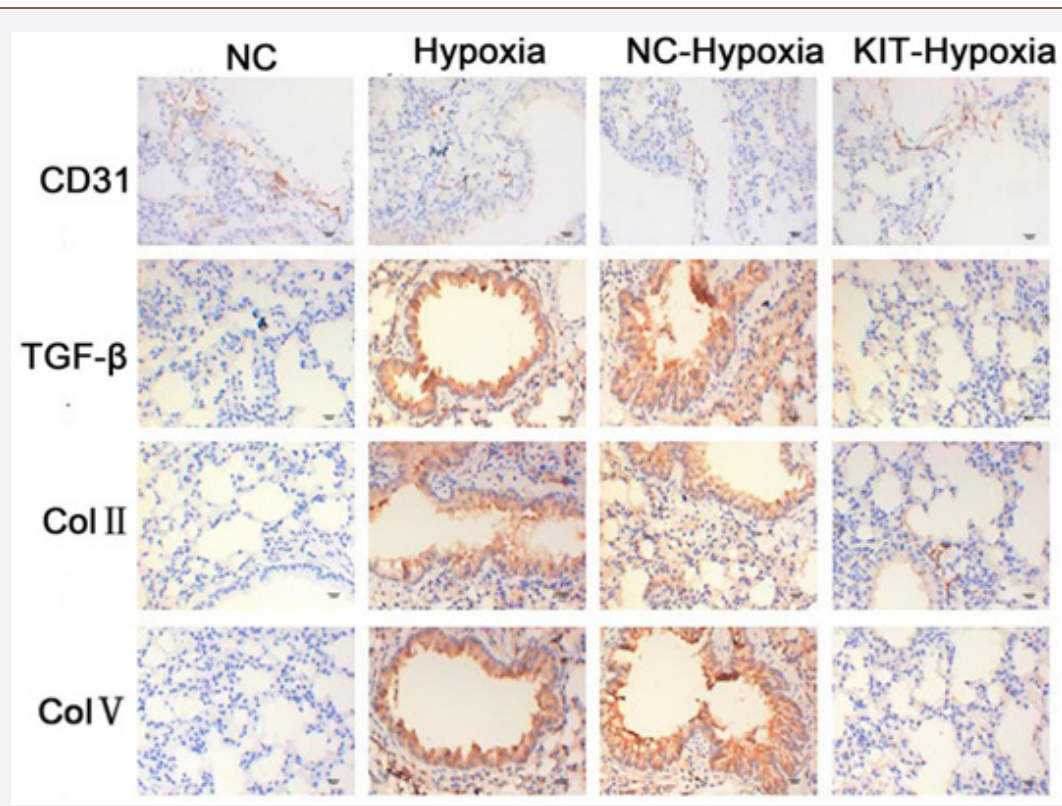

Figure 3: Immunohistochemistry analysis of CD31, TGF- $\beta, \mathrm{COL}$ II and COL V protein expression levels. The KIT + hypoxia group exhibited increased CD31 protein expression levels and significantly reduced TGF- $\beta$, COL II, and COL V protein expression levels compared with the hyperoxia group. ${ }^{*} \mathrm{P}<0.05$. Magnification, $x 400$. KIT, Tyrosine-Protein Kinase Kit; TGF- $\beta$, transforming growth factor- $\beta$; COL II, type II collagen, COL V, type $\mathrm{V}$ collagen. 
VEGF knockdown reverses functional changes in the lung caused by KIT overexpression. The results showed that compared with the NC group, the mRNA expression levels of KIT were increased in the Lv-KIT group, and VEGF expression was decreased in the same cells. In the co-transfection group, the gene expression of KIT was significantly higher compared with the sh-VEGF group; whereas, no significant difference was observed in the expression of VEGF compared with the NC group. These results suggest that VEGF is a downstream target gene of KIT (Figure 4A). Additionally, the expression levels of KIT, VEGF and Ki67 were detected in the NChypoxia, KIT-hypoxia, sh-VEGF-hypoxia and KIT + sh-VEGF-hypoxia groups using western blotting. The results showed that VEGF expression was significantly lower in the sh-VEGF hypoxia groups compared with the NC-hypoxia group, and that KIT overexpression reversed the decrease in VEGF expression induced by sh-VEGF (Figure 4B). Ki67 protein expression levels were increased in the KIT overexpression group and decreased in the sh-VEGF-hypoxia groups. VEGF silencing reversed the KIT overexpression-mediated increase in Ki67 expression in the KIT + sh-VEGF-hypoxia group (Figure 4B).

Masson's staining and immunohistochemistry were used to determine the expression of CD31 and COL II. Compared with the NC-hypoxia group, expression of collagenous fiber in the KIT overexpression groups was decreased, and VEGF silencing reversed this decrease in the KIT-hypoxia group (Figure 4C). Additionally, CD31 protein expression levels were increased in the KIT overexpression groups and sh-VEGF reduced the increase in CD31 expression levels in the KIT-hypoxia group, whereas COL II expression levels were significantly decreased in the KIT-hypoxia group and knockdown of VEGF restored COL II expression in the KIT-hypoxia group (Figure 4D). Together, these data suggest that KIT overexpression attenuated BPD-induced damage by regulating VEGF expression levels.

\section{Discussion}

BPD is a chronic pulmonary disorder of persistent respiratory distress caused by impaired alveolar and pulmonary vascular development. It is a common disorder of the respiratory system and the primary cause of death in very premature infants. BPD is characterized by alveolar simplification, abnormally reduced alveolar capillary development and early pulmonary remodeling [25]. Gene therapy is a fundamental method for treating BPD. Repairing abnormal gene expression including Thioredoxin Reductase-1 [26], miR-34a/Pdgfra [27] and Fgf10 [28] during the BPD recovery period may reduce the development of pneumonia and other complications. The results of the present study indicated that KIT overexpression in the BPD model mice may effectively prevent alveolar simplification, limited the reduction in lung angiogenesis, and inhibited disorganized and abnormal lung remodeling, thereby reducing the effects of BPD in preterm newborn mice.
To investigate the role and expression of KIT in BPD, a hyperoxiainduced newborn mouse BPD model was used in the present study which simulated the entire pathophysiological course of BPD development and progression in preterm infants [29]. At present, $75 \%$ hyperoxia is the most commonly used 02 concentration for hyperoxia lung injury in BPD experimental animal models, as it produces chronic lung injury in a short time, and providing 02 after 2 weeks resulted in the development of BPD [22]. The present study showed that providing 02 to newborn mice after 2 weeks resulted in simplification of alveolar structure, a significant reduction in the number of alveolar capillaries, pulmonary fibrosis, arrested alveolarization and other phenomena.

Studies have shown that angiogenesis during lung development is necessary for alveolar differentiation. Vasodilation is directly associated with alveolar differentiation, and its synchronization may result in the formation of an effective blood-air barrier. Preterm birth and its associated treatments may interfere with alveolar and lung vascular development, leading to the inhibition of alveolar development, reduction in the number of alveoli and capillaries, and decrease in the surface area for blood gas exchange [30]. The present study showed that KIT overexpression in the hyperoxiainduced BPD model significantly improved lung tissue structure and reduced respiratory system resistance in newborn mice by up regulating the expression of angiogenesis and the cell proliferation markers Ki67 and CD31, and down regulating the protein expression levels of markers associated with pulmonary fibrosis, including TGF- $\beta$, COL II and COL V. Together these observations confirm that KIT treatment was an effective method for reducing hyperoxia-induced BPD damage in newborn mice and suggests that KIT therapy may serve as a novel and effective clinical treatment for BPD in preterm infants.

VEGF, FGF, platelet-derived growth factor (PDGF) and Kit stimulate cellular responses through tyrosine kinase receptors [31]. Enhancement of c-Kit-R pathways results in the release of VEGF, PDGF- $\beta$ and FGF. Chou et al. [32] showed that lung development and fibrosis in BPD were accompanied by a reduction in VEGF expression, and that the administration of human mesenchymal stem cells (MSCs) improved deficient alveolarization, increased lung vascular density, reduced pulmonary fibrosis and up regulated VEGF expression [32]. Additionally, researchers have used amniotic fluid stem cell-mediated VEGF treatment in a preterm rabbit model of BPD and demonstrated that VEGF could similarly mitigate lung injury [33]. In a clinical case-controlled study, VEGF expression levels in BPD preterm infants were significantly lower compared with healthy term infants [34]. The results of the present study suggested that KIT relieved alveolar remodeling and pulmonary fibrosis in newborn BPD mice by targeting VEGF, thus, KIT acts upstream of VEGF [17]. The increase in production of iNOS as a result of hypoxia influences VEGF expression, which may 
exacerbate BPD [35]. And this has also been shown in the functional recovery experiments, where VEGF knockdown rescued the various functional changes caused by KIT overexpression.

There are several clinical hazards and unknown means of specific pathogenesis associated with BPD, therefore, clinical treatment and preventative measures are limited for treatment of BPD [36-38]. In the present study, the protective role of KIT in BPD was demonstrated and a theoretical basis for clinical prevention and treatment of BPD was established. While the samples of the present study were used up till now further research was needed to address the quantitation of the magnitude of KIT or its downstream mediators or downstream targets.

\section{Acknowledgment}

We would like to thank the staff of The Third Xiangya Hospital of Central South University for their technical support with mouse breeding and the establishment of the hyperoxia-induced BPD model, and for any other relevant technical assistance.

\section{Funding}

The presentstudy was supported byagrantfrom Guangxi Natural Science Foundation Project (grant. No. 2017GXNSFAA198075, 2017 JA10672).

\section{Availability of Data and Materials}

The datasets used and/or analyzed during the present study are available from the corresponding author on reasonable request.

\section{Authors' Contributions}

Lan Guofeng, Wang Yijin and Shi Xuekai carried out study design; Li Yunfang and Wei Qinghua performed the experiments; Shi Fenglang, Cui Qiliang and Hussnain Mirza did statistical analysis. Lan Guofeng and Shi Xuekai wrote the paper. All authors read and approved the final manuscript.

\section{Ethics Approval and Consent to Participate}

The experimental procedures and animal conditions were approved by the Medical Ethics Committee and Institutional Review Board of the Second People's Hospital of Nanning.

\section{Conflict of Interest}

The authors declare that they have no conflict of interest.

\section{References}

1. Jobe AH (2011) The new bronchopulmonary dysplasia. Current Opinion in Pediatrics 23(2): 167-172.

2. Trembath A and Laughon M (2012) Predictors of Bronchopulmonary Dysplasia. Clin Perinatol 39(3): 585-601.

3. Kobaly K, Schluchter M, Minich N, et al. (2008) Outcomes of extremely low birth weight $(<1 \mathrm{~kg})$ and extremely low gestational age ( $<28$ weeks) infants with bronchopulmonary dysplasia: effects of practice changes in 2000 to 2003. Pediatrics 121: 73-81.

4. Ho LY (2002) Bronchopulmonary dysplasia and chronic lung disease of infancy: strategies for prevention and management. Annals of the Academy of Medicine Singapore 31: 119.
5. Zhang ZH, Pan YY, Jing RS, Luan Y, Zhang L, et al. (2016) Protective effects of BMSCs in combination with erythropoietin in bronchopulmonary dysplasia-induced lung injury. Molecular Medicine Reports 14(2): 13021308.

6. Xu J, Woods CR, Mora AL, Joodi R, Brighamm KL, et al. (2007) Prevention of endotoxin-induced systemic response by bone marrow-derived mesenchymal stem cells in mice. Am J Physiol Lung Cell Mol Physiol 293(1): 131-141.

7. Rossi P, Sette C, Dolci S, Geremia R (2000) Role of c-kit in mammalian spermatogenesis. Journal of Endocrinological Investigation 23(9): 609615.

8. Wang WJ, Li HT, Yu JP, Li YM, Han XP, et al. (2018) Identification of key genes and associated pathways in KIT/PDGFRA wild-type gastrointestinal stromal tumors through bioinformatics analysis. Molecular medicine reports 18(5): 4499-4515.

9. Klug LR, Bannon AE, Javidi-Sharifi N, Town A, Flaming WH, et al. (2018) LMTK3 is essential for oncogenic KIT expression in KIT- mutant GIST and melanoma. Oncogene 38(8): 1200-1210.

10. Gebreyohannes YK, Wozniak A, Zhai ME, et al. (2018) Robust Activity of Avapritinib, Potent and Highly Selective Inhibitor of Mutated KIT, in Patient-derived Xenograft Models of Gastrointestinal Stromal Tumors. Clin Cancer Res 25(2): 609-618.

11. Abman SH (2010) Impaired vascular endothelial growth factor signaling in the pathogenesis of neonatal pulmonary vascular disease. Humana Press.

12. Askie LM, Ballard RA, Cutter GR, Dani C, Elbourne D, et al. (2011) Inhaled nitric oxide in preterm infants: an individual-patient data meta-analysis of randomized trials. Pediatrics 128(4): 729-739.

13. Thébaud B, Ladha F, Michelakis ED, Sawicka M, Thurston G, et al. (2005) Vascular endothelial growth factor gene therapy increases survival, promotes lung angiogenesis, and prevents alveolar damage in hyperoxia-induced lung injury: evidence that angiogenesis participates in alveolarization. Circulation 112: 2477-2486.

14. Bhatt J, Amin SB, Chess PR, Watkins RH, Maniscalco WM (2000) Expression of Vascular Endothelial Growth Factor and Flk-1 in Developing and Glucocorticoid-Treated Mouse Lung. Pediatr Res 47(5): 606-613.

15. Simpson A, Custovic A, Tepper R, Graves P, Stern DA, et al. (2012) Genetic Variation in Vascular Endothelial Growth Factor-A and Lung Function. Am J Respir Crit Care Med 185(11): 1197-1204.

16. Aslam M, Baveja R, Liang OD, Fernandez-Gonzalez A, Lee C, et al. (2009) Bone Marrow Stromal Cells Attenuate Lung Injury in a Murine Model of Neonatal Chronic Lung Disease. Am J Respir Crit Care Med 180(11): 1122-1130.

17. Feng ZC, Popell A, Li J, Silverstein J, Oakie A, et al. (2015) c-Kit Receptor Signaling Regulates Islet Vasculature, beta-Cell Survival, and Function In Vivo. Diabetes 64(11): 3852-3866.

18. Hendricks-Munoz KD, Xu J, Voynow JA (2018) Tracheal aspirate VEGF and sphingolipid metabolites in the preterm infant with later development of bronchopulmonary dysplasia. Pediatr Pulmonol 53(8): 1046- 1052.

19. Mahlman M, Huusko JM, Karjalainen MK, et al. (2015) Genes Encoding Vascular Endothelial Growth Factor A (VEGF-A) and VEGF Receptor 2 (VEGFR-2) and Risk for Bronchopulmonary Dysplasia. Neonatology 108: 53-59.

20. Procianoy RS, Hentges CR and Silveira RC (2016) Vascular Endothelial Growth Factor/Placental Growth Factor Heterodimer Levels in Preterm Infants with Bronchopulmonary Dysplasia. Am J Perinatol 33: 480- 485.

21. Mei Y, Chen C, Dong H, Zhang W, Wang Y, et al. (2018) Treatment of Hyperoxia-Induced Lung Injury with Lung Mesenchymal Stem Cells in Mice. Stem Cells Int 2018: 5976519.

22. Hansmann G, Fernandezgonzalez A, Aslam M, Vitali SH, Martin T, et al. (2012) Mesenchymal Stem Cell-Mediated Reversal of Bronchopulmonary 
Dysplasia and Associated Pulmonary Hypertension. Pulmonary Circulation 2(2): 170-181.

23. Lui K, Lloyd J, Ang E, Rynn M, Gupta JM (2015) Early changes in respiratory compliance and resistance during the development of bronchopulmonary dysplasia in the era of surfactant therapy. Pediatric Pulmonology 30(4): 282-290.

24. Mižíková I, Pfeffer T, Nardiello C, Surate Solaligue DE, Steenbock H, et al. (2018) Targeting transglutaminase 2 partially restores extracellular matrix structure but not alveolar architecture in experimental bronchopulmonary dysplasia. FEBS J 285(16): 3056-3076.

25. Villela LD, Mdbb M, Scs GJ, de Abranches AD, Fvm S, et al. (2018) Body composition in preterm infants with intrauterine growth restriction: a cohort study. J Perinat Med 46(7): 804-810.

26. Wall SB, Wood R, Dunigan K, Li Q, Li R, et al. (2019) Thioredoxin Reductase-1 Inhibition Augments Endogenous Glutathione-Dependent Antioxidant Responses in Experimental Bronchopulmonary Dysplasia. Oxid Med Cell Longev 2019: 7945983.

27. Ruiz-Camp J, Quantius J, Lignelli E, Arndt PF, Palumbo F, et al. (2019) Targeting miR-34a/Pdgfra interactions partially corrects alveologenesis in experimental bronchopulmonary dysplasia. EMBO Mol Med 11(3): e9448.

28. Chao CM, Moiseenko A, Kosanovic D, Rivetti S, Agha EE, et al. (2018) Impact of Fgf10 deficiency on pulmonary vasculature formation in a mouse model of bronchopulmonary dysplasia. Hum Mol Genet 28(9): 1429-1444.

29. Yin J, Wang X, Zhang L, Wang X, Liu H, et al. (2018) Peptidome analysis of lung tissues from a hyperoxia-induced bronchopulmonary dysplasia mouse model: Insights into the pathophysiological process of bronchopulmonary dysplasia. Journal of Cellular Physiology 233(10): 7101-7112.

30. Desch AN, Gibbings SL, Goyal R, Kolde R, Bednarek J, et al (2016) Flow Cytometric Analysis of Mononuclear Phagocytes in Nondiseased Human
Lung and Lung-Draining Lymph Nodes. Am J Respir Crit Care Med 193(6): 614-626.

31. Mishra A, Mishra SC, Tripathi AM and Pandey A (2018) Clinical correlation of molecular (VEGF, FGF, PDGF, c-Myc, c-Kit, Ras, p53) expression in juvenile nasopharyngeal angiofibroma. Eur Arch Otorhinolaryngol 275(11): 2719-2726.

32. Chou HC, Li YT and Chen CM (2016) Human mesenchymal stem cells attenuate experimental bronchopulmonary dysplasia induced by perinatal inflammation and hyperoxia. Am J Transl Res 8(2): 342-353.

33. Jiménez J, Lesage F, Richter J, Nagatomo T, Salaets T, et al. (2018) Upregulation of Vascular Endothelial Growth Factor in Amniotic Fluid Stem Cells Enhances Their Potential to Attenuate Lung Injury in a Preterm Rabbit Model of Bronchopulmonary Dysplasia. Neonatology 113(3): 275-285.

34. Procianoy RS, Hentges CR, Silveira RC (2015) Vascular Endothelial Growth Factor/Placental Growth Factor Heterodimer Levels in Preterm Infants with Bronchopulmonary Dysplasia. Amer J Perinatol 33(5): 480485.

35. Syed MA, Rayman CW, Homer RJ, Bhandari V (2016) Role of Nitric Oxide Isoforms in Vascular and Alveolar Development and Lung Injury in Vascular Endothelial Growth Factor Overexpressing Neonatal Mice Lungs. PLOS ONE 11(1): e014758.

36. Sakurai R, Li Y, Torday JS, Rehan VK (2011) Curcumin augments lung maturation, preventing neonatal lung injury by inhibiting TGF- $\boldsymbol{\beta}$ signaling. Am J Physiol Lung Cell Mol Physiol 301(5): 721-730.

37. Koh Y (2014) Update in acute respiratory distress syndrome. Journal of Intensive Care 2(1): 2.

38. Hendricks Muñoz KD, Xu J, Voynow JA (2018) Tracheal aspirate VEGF and sphingolipid metabolites in the preterm infant with later development of bronchopulmonary dysplasia. Pediatr Pulmonol 53(8): 1046-1052. 